\title{
Pulmonary artery sling diagnosed and corrected in an 11-year-old boy with refractory pulmonary infections and childhood-onset asthma
}

\author{
Ireneusz Haponiuk ${ }^{1,2}$, Maciej Chojnicki ${ }^{1}$, Konrad Paczkowski ${ }^{1}$, Jolanta Zabłocka ${ }^{1}$, Mariusz Steffens ${ }^{1}$, \\ Marta Paśko-Majewska1 ${ }^{1}$, Wiktor Szymanowicz ${ }^{1}$, Katarzyna Gierat-Haponiuk ${ }^{2}$

\begin{abstract}
${ }^{1}$ Department of Pediatric Cardiac Surgery, St. Adalbertus Hospital Copernicus, Gdansk-Zaspa, Poland
2Department of Physiotherapy, Faculty of Rehabilitation and Kinesiology, Gdansk University of Physical Education and Sport, Gdansk, Poland
\end{abstract}

Pulmonary artery sling (PAS) is a congenital anomaly in which a pulmonary artery branch crosses respiratory tract organs (trachea or main bronchi) from the opposite side with their overlapping and airway obstruction. The defect is usually diagnosed in early infancy because of an evident clinical manifestation related more to respiratory than circulatory problems [1]. This rare anomaly is usually identified in the settings of complex congenital anomalies, although it can occur as an isolated problem or partial form with usually coexisting tracheal stenosis [2].

We report an adolescent patient with PAS that was diagnosed late after recurrent and refractory pulmonary infections, and symptoms mimicking childhood-onset asthma. Surgical correction with anatomic reconstruction of the anatomic position of the main pulmonary artery with its bifurcation into the natural course was planned as a procedure of choice.
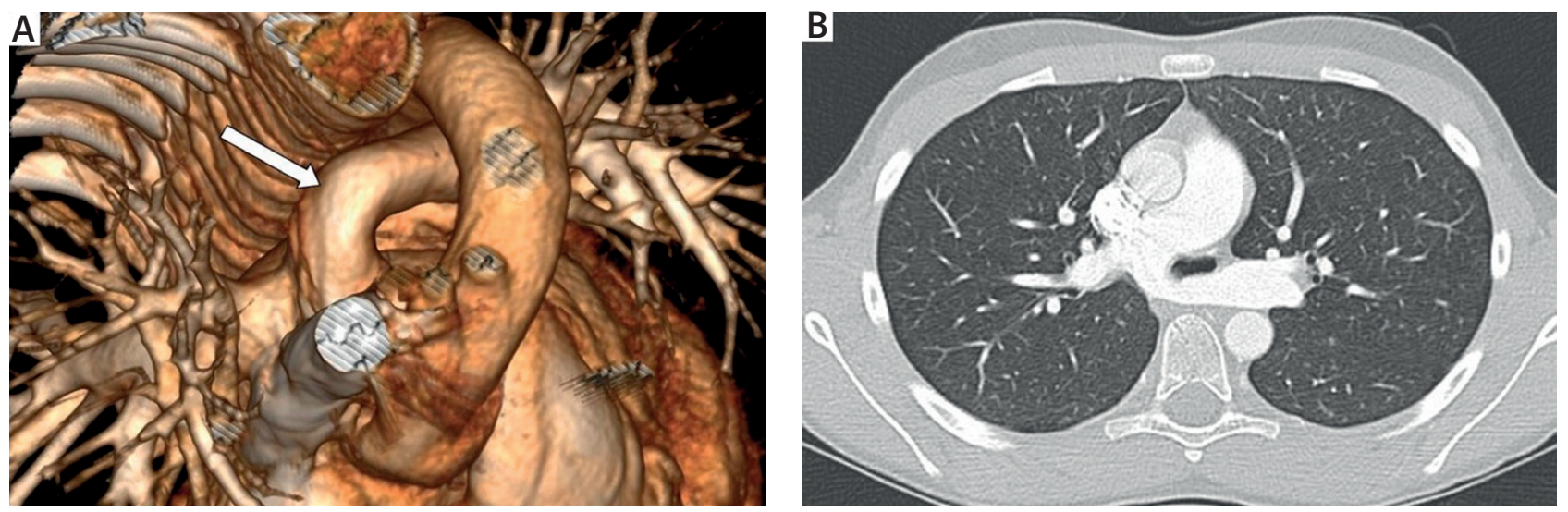

Fig. 1. A - Angio-CT scan with an anatomic reconstruction. The arrow indicates the retrotracheal course of the anomalously originated left pulmonary artery (LPA) from the right pulmonary artery (PA sling). B - Angio-CT: great care was taken to exclude evidence of tracheal malacia in the region of chronic impression by the pathological course of the LPA

Address for correspondence: Ireneusz Haponiuk MD, PhD, Associate Prof., Department of Pediatric Cardiac Surgery, St. Adalbertus Hospital Copernicus, 50 John Paul II Av., 80-462 Gdansk, Poland, phone: +48 5876848 81, e-mail: ireneusz_haponiuk@poczta.onet.pl Received: 8.06.2018, accepted: 31.08.2018. 

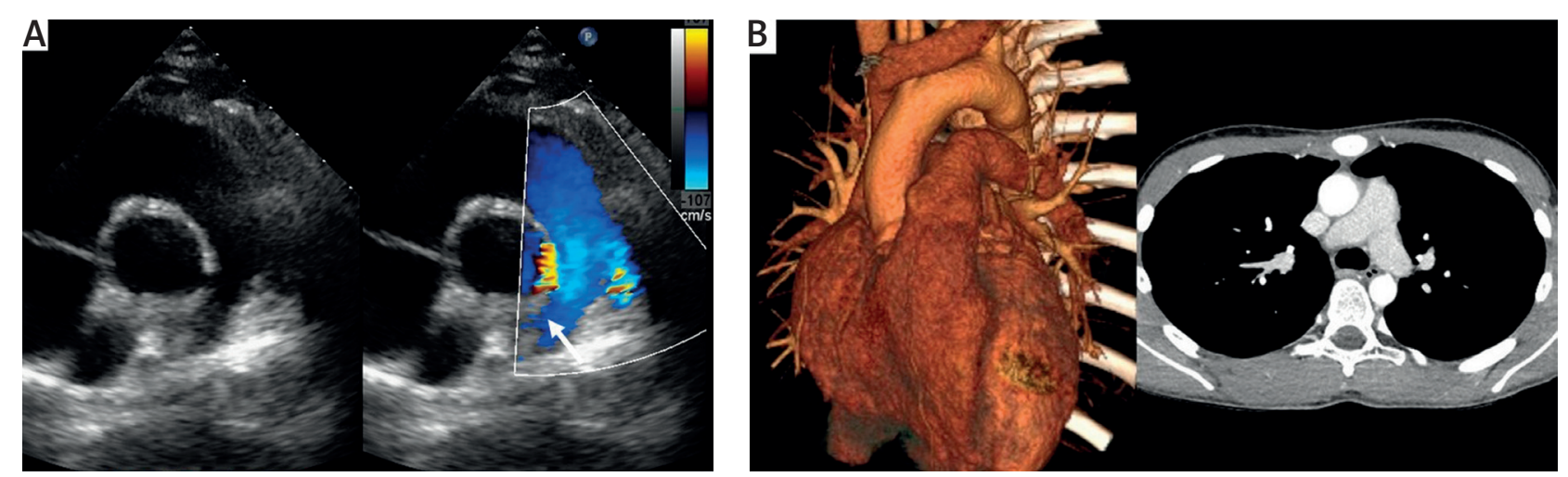

Fig. 2. A - Postoperative transthoracic echocardiography - short time after surgery: natural antegrade flow from right ventricle and pulmonary trunk was directed to still widened right pulmonary artery (RPA - arrow). B - Control angio-CT performed 2 years after the reconstruction: any obstruction, underdevelopment or hypoplasia of the left pulmonary artery was excluded

nary artery (PA) trunk directly to the right pulmonary artery (RPA), while the position of the LPA was difficult to visualize with carefully repeated classic TTE.

He was finally referred for surgical correction of the defect. The procedure was performed with normothermic extracorporeal circulation on a beating heart. Intraoperatively an extended PA trunk and oversized RPA were identified. After meticulous preparation an anomalous origin of the LPA arising from the extended proximal RPA crossing the trachea from its posterior side, directly above the tracheal bifurcation, was found. Initially the LPA was detached from the RPA, and its bed was meticulously dissected free behind the trachea. Great efforts were made to prevent uncontrolled bleeding, tearing, electrocautery damage or puncture of the potentially thinned wall of the trachea. The distal part of the LPA was initially identified as well in the left lung hilum, and then dissected free proximally in the direction of its postero-tracheal course. After the detachment and the transfer for the left side an end-to-side anastomosis was performed between the main PA and the LPA in front of the trachea. Finally the new anastomosis was followed by the creation of a pericardial window just below the left vagal and phrenic nerves. Then the natural forward flow from the right ventricle directed independently into both lungs was confirmed by epicardial echocardiography, without an intraoperative picture of local stenosis, turbulent flow or closure (Fig. 2 A). Conflict with other vital organs such as the tracheal tract, aorta and the esophagus was avoided. The diaphragmatic respiratory function was undisturbed as well.

The abnormalities of systemic as well as pulmonary arteries could cause misleading pulmonary symptoms, usually manifested in the early neonatal period. Nevertheless, congenital vascular anomalies in the mediastinum in older children and adolescents suffering from recurrent or chronic respiratory problems should also be considered, regardless of the age.

The clinical consequences of an impression of pulmonary arteries seem to be less aggressive when compared to the course of anomalous systemic arteries. The reasons probably derive from lower pressure and less pulsation of the pulmonary bed, which causes lower local impression and damaging forces over surrounding organs. Therefore every diagnosis of pulmonary arteries' abnormal course, as in the present patient with PA sling, should be referred for surgical correction after meticulous diagnosis [3,4].

Following rare literature reports, pulmonary artery sling promotes local complications related to the chronic impression for the respiratory tract [4]. The low-pressure pulmonary bed gives the chance to prevent the most important and irreversible complications known as tracheal cartilages malacia. Finally the presented correction for the anatomic position improved pulmonary flow and does not interfere with the natural potential to grow according to the age of our patient (Fig. 2 B). The most important argument was an improvement of the general status of the boy as well as the resolution of his chronic infections and pediatric pulmonary obstructive symptoms.

Simple discontinuation of anomalous LPA in a pulmonary artery sling defect with subsequent reconstruction of the pulmonary trunk with naturally positioned both main branches preserved competent pulmonary blood flow without the risk of hypoperfusion and local impression.

Despite the teenage age of the patient referred for the correction of PAS, there were no diagnostic, intraoperative or clinical findings of local, or long-lasting general complications related to late diagnosis of the congenital pulmonary artery defect.

\section{Disclosure}

The authors report no conflict of interest.

\section{References}

1. Inui T, Yamada H, Hida N, Terashima H, Saito T, Hizawa N. A case of pulmonary artery sling misdiagnosed as refractory asthma for 20 years. Clin Case Rep 2017; 5: 863-866.

2. Nagatomo Y, Meneuchi J, Watanabe M, Joo K, Ochiai Y. Repair of partial pulmonary artery sling in a symptomatic adolescent. Ann Thorac Surg 2017; 104: e289.

3. Strzelczuk-Judka L, Jończyk-Potoczna K, Mroziński B, Mrówczyński W. Zastosowanie angiografii tomografii komputerowej u dzieci z koarktacją aorty doświadczenia własne. Folia Cardiol 2014; 9: 228-236.

4. Woods RK, Sharp RJ, Holcomb GW $3^{\text {rd }}$, Snyder CL, Lofland GK, Ashcraft KW, Holder TM. Vascular anomalies and tracheoesphageal compression: a single institution's 25-year experience. Ann Thorac Surg 2011; 72: 434-439. 